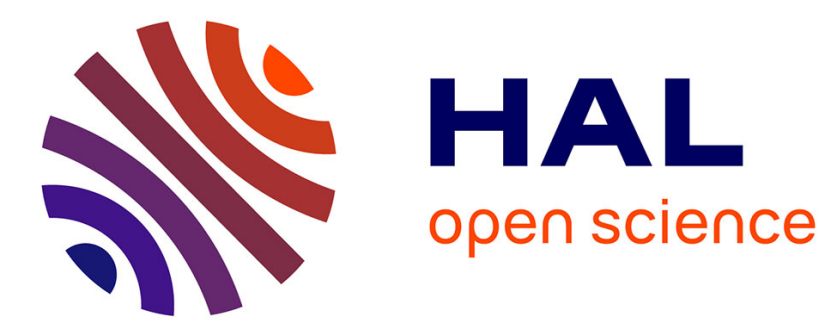

\title{
Recherche sur les champs magnétiques intenses hyperconducteurs pour des expériences de physique des particules
}

\author{
J.P. Massue, J. Royer, H. Éberlé, R. Oppel, H. Braun
}

\section{To cite this version:}

J.P. Massue, J. Royer, H. Éberlé, R. Oppel, H. Braun. Recherche sur les champs magnétiques intenses hyperconducteurs pour des expériences de physique des particules. Revue de Physique Appliquée, 1969, 4 (2), pp.230-232. 10.1051/rphysap:0196900402023000 . jpa-00243235

HAL Id: jpa-00243235

https://hal.science/jpa-00243235

Submitted on 1 Jan 1969

HAL is a multi-disciplinary open access archive for the deposit and dissemination of scientific research documents, whether they are published or not. The documents may come from teaching and research institutions in France or abroad, or from public or private research centers.
L'archive ouverte pluridisciplinaire HAL, est destinée au dépôt et à la diffusion de documents scientifiques de niveau recherche, publiés ou non, émanant des établissements d'enseignement et de recherche français ou étrangers, des laboratoires publics ou privés. 


\title{
REGHERGHE SUR LES GHAMPS MAGNÉTIQUES INTENSES HYPERGONDUGTEURS POUR DES EXPÉRIENGES DE PHYSIQUE DES PARTIGULES
}

\author{
J. P. MASSUE, J. ROYER, H. ÉBERLÉ, R. OPPEL et H. BRAUN, \\ Centre de Recherches Nucléaires, Strasbourg.
}

\begin{abstract}
Résumé. - On décrit la construction de bobines pulsées hyperconductrices. La durée de l'impulsion est en moyenne de l'ordre de la milliseconde, le diamètre intérieur de l'ordre de $10 \mathrm{~mm}$ et les champs de l'ordre de $400 \mathrm{kG}$.

Abstract. - We describe the construction of pulsed hyperconducting coils. The time of a pulse is on the average of the order of a ms. The internal diameter is of the order of $10 \mathrm{~mm}$ and the fields of about $400 \mathrm{kG}$.
\end{abstract}

I. Introduction. - Ge travail a été entrepris dans un double but $:$ i) étudier la courbure des trajectoires dans les émulsions ionographiques, afin de $: a$ ) disposer d'un paramètre supplémentaire; $b$ ) déterminer, de façon rapide, le signe de la charge des particules, par exemple: réaction de double échange de charge $\pi^{+}-\pi^{-}$; ii) mettre au point des faisceaux d'hypérons, par exemple : faisceau de $\Xi$ pour l'obtention d'hyperfragments doubles.

II. Principe. - Nous avons calculé et construit des bobines constituées par des enroulements de fil de cuivre isolé au polyglass en bande et maintenus mécaniquement par des flasques en fibre de verre. Ces bobines sont refroidies à la température de l'azote liquide. L'équilibre thermique atteint, nous déchargeons une batterie de condensateurs dans ces bobines. Les essais ont été faits sur deux types de bobines : des simples et des doubles; nous avons utilisé deux bancs de condensateurs dont les caractéristiques étaient : i) à Strasbourg au C.R.N. : $c=3900 \mu \mathrm{F}$, $V_{\max }=4000 \mathrm{~V}$; ii) au C.E.R.N. à Genève : $c \stackrel{\max }{=} 75000 \mu \mathrm{F}, V_{\max }=3000 \mathrm{~V}$. Nous avons calculé nos bobinages en donnant en général à nos bobines une self $L=100 \mu \mathrm{H}$, le diamètre intérieur variant de 7 à $20 \mathrm{~mm}$ et le diamètre extérieur variant de 50 à $100 \mathrm{~mm}$. Pour les bobines doubles, nous avons choisi la distance entre deux bobines $D$ égale au diamètre intérieur $2 a_{1}$ de celle-ci.

III. Résultats. - 1) Résultats obtenus avec le banc de condensateur du G.E.R.N. à Genève : $c=75000 \mu \mathrm{F}$, $V_{\text {max }}=3000 \mathrm{~V}$.

Nous donnons les résultats ( $f g .1$ ) pour la bobine $\mathbf{M}_{6}$ de diamètre intérieur $10 \mathrm{~mm}$. La courbe a) donne des résultats obtenus avec le banc du C.E.R.N.; la courbe b) donne des résultats avec le banc de Strasbourg. Avec le banc de $75000 \mu \mathrm{F}$, la courbe présente un maximum suivi d'une partie décroissante conduisant à la détérioration de la bobine.
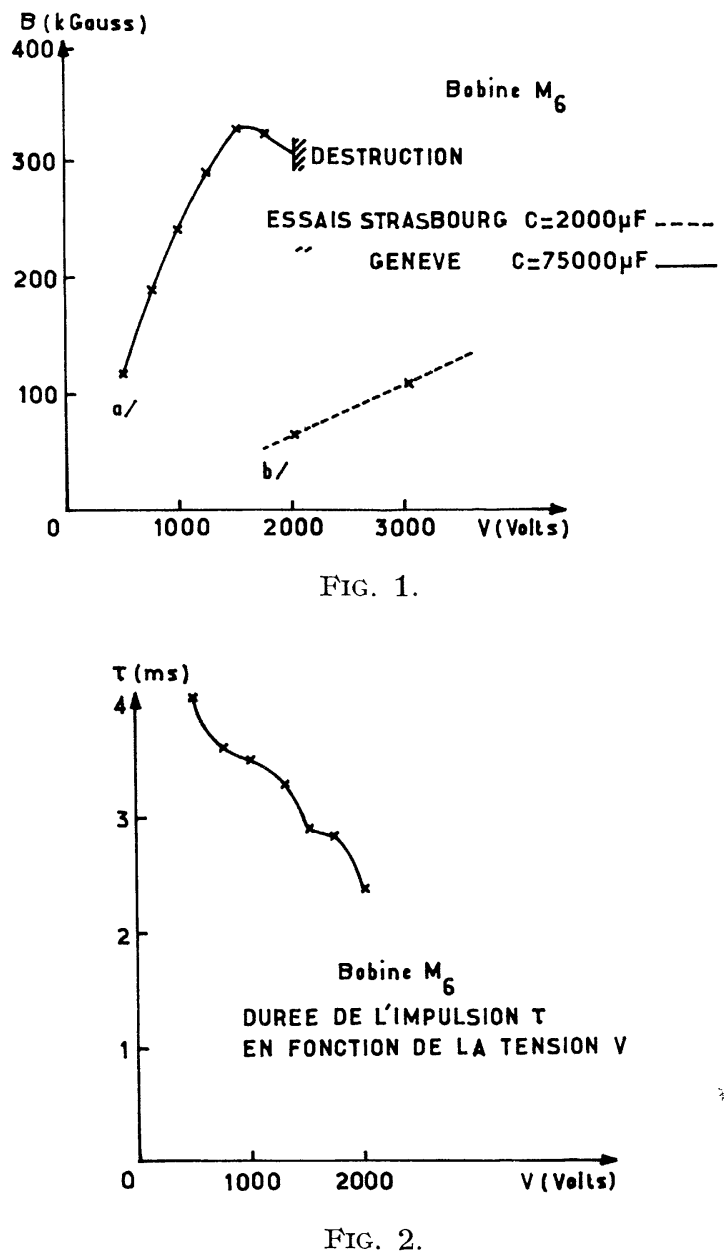

D'autre part, lors de ces essais, nous avons tracé la durée de l'impulsion en fonction de la tension ( fig. 2) pour la bobine $\mathbf{M}_{6}$. Dans ce cas, la durée de l'impulsion 
décroît rapidement quand la tension croît. On peut penser que la capacité $c=75000 \mu \mathrm{F}$ était trop importante pour nos bobines, ce qui conduit à un passage de courant important pendant ces temps trop longs dans le conducteur. On a donc effectué une deuxième série d'essais avec un banc de capacité plus faible.

La figure 3 montre les bobines $M_{6}$ et $M_{7}$.

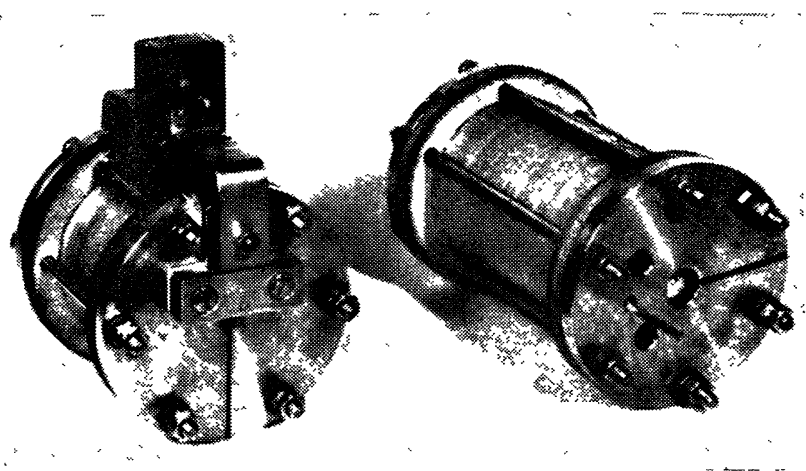

FIG. 3.
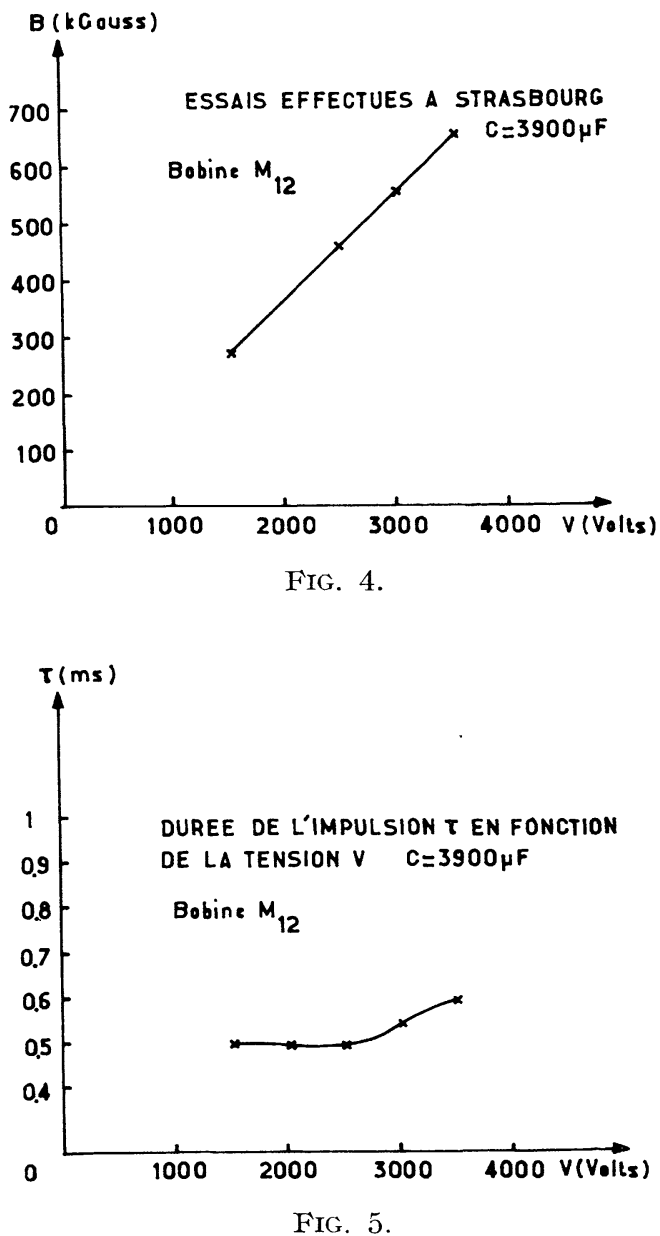

2) Résultats obtenus au C.R.N. de Strasbourg : Nous avons travaillé avec une bobine $\mathrm{M}_{12}$ dont la self était $L=56 \mu \mathrm{H}$, le diamètre intérieur $8 \mathrm{~mm}$, le diamètre extérieur $50 \mathrm{~mm}$ et la longueur $50 \mathrm{~mm}$. Nous

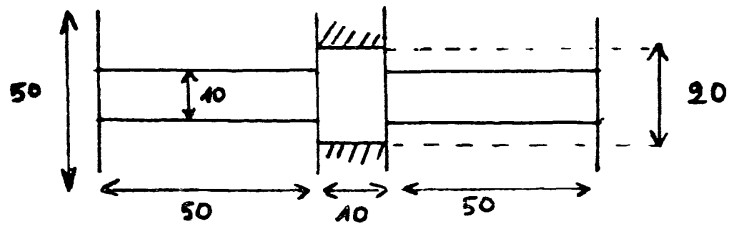

FIG. 6.

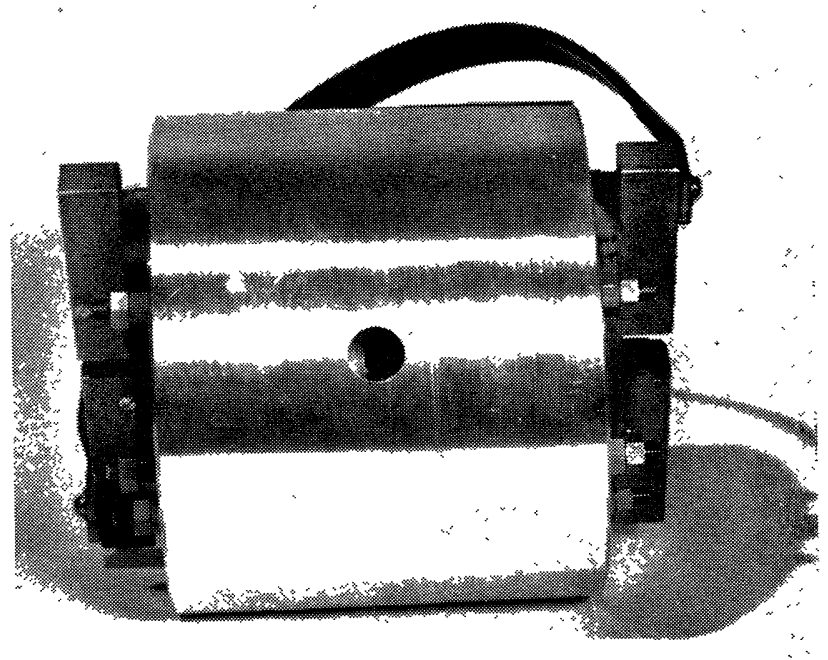

FIG. 7.

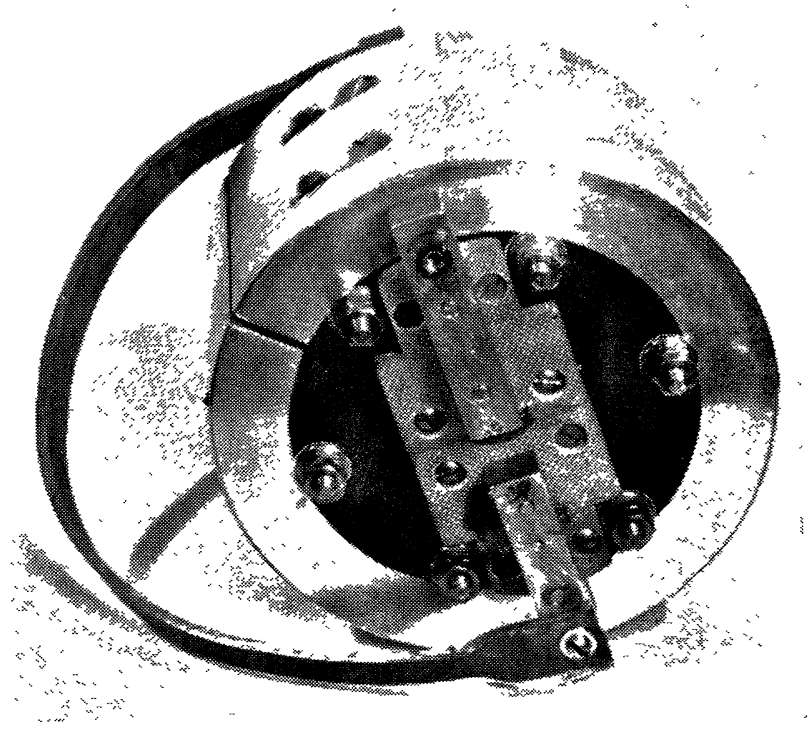

Fıง̂. 8. 
avons fait un essai avec un banc de $3900 \mu \mathrm{F}$. Les résultats sont donnés par les figures 4 et 5 . Pour vérifier la résistance mécanique de cette bobine, nous avons pulsé celle-ci en automatique à $300 \mathrm{kG}$ avec une période de 30 s pendant 250 pulses, nous n'avons noté aucune défectuosité mécanique.

Nous avons ensuite essayé une bobine double $\mathrm{M}_{13}$ dont les dimensions en $\mathrm{mm}$ sont indiquées sur le schéma de la figure 6 .

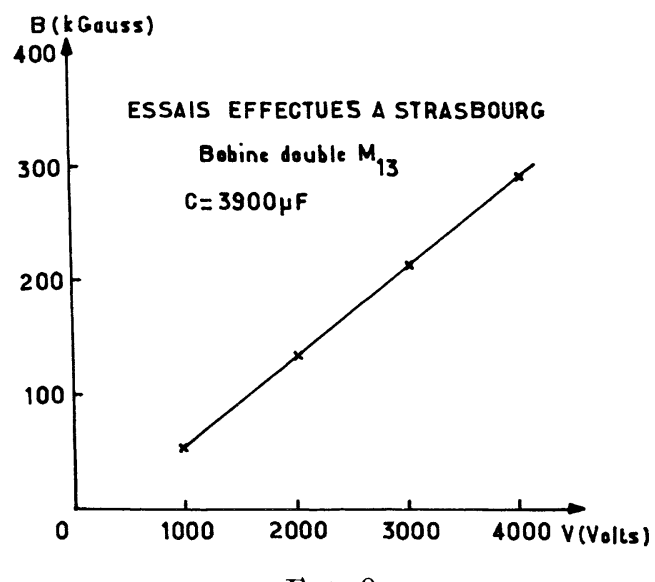

FIG. 9.

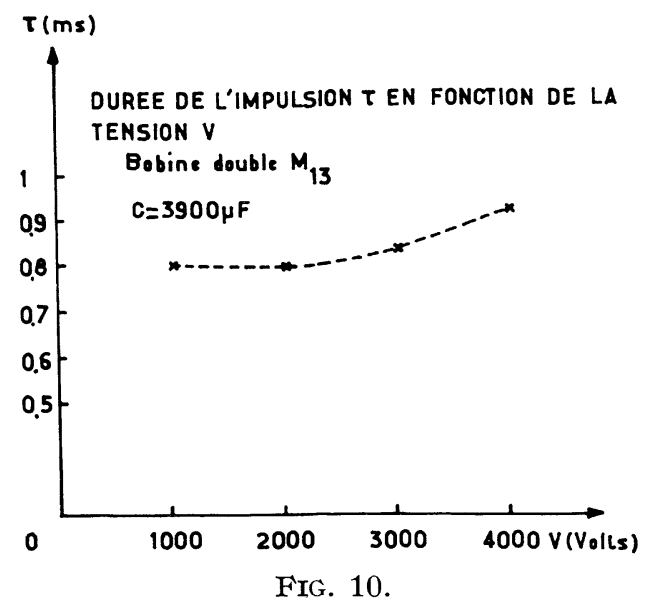

Les figures 7 et 8 nous montrent la bobine vue de son côté et perpendiculairement à son axe.

Les résultats sont donnés par les figures 9 et 10 , nous n'avons appliqué qu'un banc de $3900 \mu \mathrm{F}$ sur l'ensemble de deux bobines, les bobines fonctionnant en série. Donc, nous pourrons augmenter le champ, mais ces premiers essais étaient surtout pour contrôler la résistance mécanique de ces bobines doubles : le test a été positif. 